\title{
Suppressing Motion Artefacts in MRI using an Inception-ResNet network with Motion Simulation Augmentation
}

Kamlesh Pawar ${ }^{1,2^{*}}$, Zhaolin Chen ${ }^{1}$, N. Jon Shah ${ }^{1,3}$, Gary F. Egan ${ }^{1,2}$

1. Monash Biomedical Imaging, Monash University, Melbourne, Australia

2. School of Psychological Sciences, Monash University, Melbourne, Australia

3. Institute of Medicine, Research Centre Jülich, Jülich, Germany

Running Head: Suppressing Motion Artefacts in MRI using an Inception-Resnet

Journal: NMR in Biomedicine

* Corresponding author:

\author{
Name Kamlesh Pawar \\ Department Monash Biomedical Imaging \\ Institute Monash University \\ Address 770 Blackburn Road, Notting Hill, VIC-3168, Australia \\ E-mail kamlesh.pawar@monash.edu
}

This is the author manuscript accepted for publication and has undergone full peer review but has not been through the copyediting, typesetting, pagination and proofreading process, which may lead to differences between this version and the Version of Record. Please cite this article as doi: $10.1002 / \mathrm{nbm} .4225$

This article is protected by copyright. All rights reserved. 
Manuscript word count: 4300

Abstract word count: 250

\begin{abstract}
The suppression of motion artefacts from MR images is a challenging task. The purpose of this paper was to develop a standalone novel technique to suppress motion artefacts in MR images using a data-driven deep learning approach. A simulation framework was developed to generate motion-corrupted images from motion-free images using randomly generated motion profiles. An Inception-ResNet deep learning network architecture was used as the encoder and was augmented with a stack of convolution and upsampling layers to form an encoder-decoder network. The network was trained on simulated motion-corrupted images to identify and suppress those artefacts attributable to motion. The network was validated on unseen simulated datasets and real-world experimental motion-corrupted in vivo brain datasets. The trained network was able to suppress the motion artefacts in the reconstructed images, and the mean SSIM increased from 0.9058 to 0.9338 . The network was able to suppress the motion artefacts from the real-world experimental dataset and the mean SSIM increased from 0.8671 to 0.9145 . The motion correction of the experimental datasets demonstrated the effectiveness of the motion simulation generation process. The proposed method successfully removed motion artefacts and outperformed an iterative entropy minimization method in terms of the SSIM index and normalised root mean squared error, which were $5-10 \%$ better for the proposed method. A novel, data-driven motion correction technique has been developed that can suppress motion artefacts from motion-corrupted MR images. The proposed technique is a standalone, post-processing method that does not interfere with data acquisition or reconstruction parameters, thus making it suitable for routine clinical practice.
\end{abstract}

Keywords: Deep learning, MR motion correction, MR image reconstruction 


\section{INTRODUCTION}

Magnetic resonance imaging (1) is an established medical imaging modality used to produce detailed images of different tissue types. However, despite its advantages, image acquisition times can be long, and any patient motion during scanning causes artefacts in the resultant MR images (2). Since the advent of MRI, the removal of motion artefacts has continued to be an unresolved problem for most clinical applications. In particular, motion artefacts present significant difficulties when scanning paediatric, elderly, claustrophobic and stroke patients. Apart from patient discomfort, motion artefacts also result in financial losses (3) for imaging sites. A number of methods can be used to correct for motion artefacts, the most commonly used ones being rigid motion correction techniques. These techniques can be broadly classified into three categories: navigator sequence-based approaches, external motion detection devices and data-driven approaches.

The navigator-based approaches involve inserting k-space, or image navigators, into the imaging sequences. The navigators are inserted within the sequence at different time points and are compared with a reference to estimate motion parameters. The parameters can subsequently be used to perform either prospective or retrospective motion correction. The first navigator sequence to estimate in-plane linear motion was proposed by Ehman et. al. (4) and involves acquiring the zeroth phase encode in both the $\mathrm{x}$ and $\mathrm{y}$ directions. The phase difference between navigators at different time points provides a measure of motion in the $\mathrm{x}$ and y directions. However, this technique only compensates for translation motion. An alternative technique, known as PROPELLER (5), is a self-navigation technique in which a 
number of straight lines called blades are acquired at different angles to cover the whole kspace. The acquisition of blades at various angles results in the acquisition of the centre of kspace multiple times, and the points that are sampled multiple times are used as navigators to extract rotation and translation information. A limitation of the PROPELLER technique is that it can only provide in-plane motion parameters. This limitation is, however, not specific to PROPELLER and is a limitation found in all 2D imaging sequences as the through-plane motion disrupts signals from different slices.

Another technique that enables compensation for linear motion, based on the acquisition of data on a Cartesian grid, is EPIK (6). EPIK is an interleaved technique that acquires the central line of k-space in each shot and therefore enables motion correction along a single axis. Application of EPIK to diffusion imaging with motion correction has been demonstrated in (7). Multiple implementations of the use of k-space navigators to estimate motion parameters in all six degrees of freedom have been developed (8-13) along with image navigators (14-17) for 3D imaging sequences. Despite the advantages afforded by EPIK, its implementation requires all navigator sequences to be carefully designed, resulting in an inevitable increase in acquisition time and a reduction in SNR. Constraints are also imposed on protocol parameters, including TR/TE/TI. Recently, a novel approach for motion correction using a spherical navigator based on a Lissajous trajectory has been demonstrated in a proof of concept study. Here, an isotropic translational accuracy of $0.2 \mathrm{~mm}$ and a rotation estimate of 0.5 degrees was demonstrated. The Lissajous navigator has only modest gradient demands, covers the poles of the k-space sphere and acquires data in approximately $5 \mathrm{~ms}$ (18).

External device-based approaches involve an estimation of the motion by using an external device, such as an optical camera, that tracks a marker placed on the patient (19-23) or by using field probes that measure the magnetic field perturbation due to motion $(24,25)$. The major limitations of external device techniques are that they involve additional cost, require calibration, and the use of mounting markers on the patient may cause patient discomfort and potential patient compliance issues, making them difficult for clinical usage.

Data-driven approaches to motion correction estimate and correct motion directly from the images. Entropy focus criteria $(26,27)$ techniques belong to the category of datadriven approaches which were used for retrospective motion correction. These approaches 
correct motion artefacts by minimizing image entropy and do not require additional navigators. However, the entropy focus criteria method relies on computationally demanding iterative algorithms.

Recently, convolutional neural networks (CNNs) have been used in MRI for image reconstruction from undersampled data (28-34) and for MR image analysis (35). The basic operation in CNNs is the convolution operation. Convolution operations are widely used in image processing applications, such as image segmentation, edge detection, image denoising, Gaussian blurring, and median filtering, to name a few. For instance, in order to remove noise from images, a simple averaging kernel for convolution can be used, which is equivalent to low pass filtering. In these applications, the convolution kernels are handengineered using prior knowledge of the imaging process, noise statistics, and the nature of the artefacts. However, algorithms for such applications, including image de-noising and segmentation, are being rapidly replaced by deep learning (31) approaches.

The use of CNNs to detect motion (36) or to correct (37-43) motion artefacts has been explored in previous studies (36-43). The concept of using CNN for end-to-end motion correction was first presented in our early work, published as a conference abstract (42). Similar work, including (43), applied CNN to individual patches of 2D spin echo images to correct for simulated motion artefacts. In (37), CNN was used as a pre-processing block to improve the motion correction physics model, and in (38), it was used as a regularizer for iterative model-based motion correction. A 3D patch-based approach using a conditiongenerative adversarial network was proposed in (40). A feasibility study of retrospective motion correction using CNNs for rigid and non-rigid motion correction in 2D imaging was presented in (41). Most of the methods (37, 38, 41, 43) applied the CNN approach to motion correction in 2D imaging, some methods $(38,43)$ applied CNNs on small patches of 2D images, while other methods $(37,38)$ used a CNN to improve the motion-based model. Motion simulation for 3D images was also performed in (40) and the authors tested their network in both simulated and experimental datasets. However, the 3D images in (40) were from a FLASH sequence at 7T with an extremely narrow field of view in both the sagittal and coronal views and the motion simulation was limited to only 2-3 motion events during the scan, which may not be a realistic estimate. 
The ringing, blurring and ghosting artefacts caused by motion can be suppressed with convolution operations. However, a set of convolution kernels that can be used to remove motion artefacts may not be analytically determined due to the random nature of the motion that causes the artefacts. The objective of this study was to simulate motion-corrupted images and subsequently use them to train a deep learning network that could learn appropriate convolution kernels to identify and correct for artefacts arising as a result of motion. We leverage the power of data-driven deep learning methods to determine sets of convolution kernels that can efficiently remove artefacts arising due to motion. We focus on the widely used 3D T1 MPRAGE images, where motion artefacts are often observed at 3T MRI. Extensive motion simulation covering the whole head in the three orthogonal views was performed. This paper describes:

1. The application of multi-resolution Inception ResNet as an encoder which consists of different convolutional kernel sizes at each resolution scale to capture both local and global motion artefacts.

2. A method to design and simulate realistic motion curves to capture both abrupt motion and gradual motion of the real-world scenarios.

3. Training and validation of deep learning models with a large dataset containing 1572 subjects for training and 384 subjects for validation. The training and validation set consisted of approximately 235K and 57K 2D slices respectively.

Here, we have substantially improved our previous method (42) and have used a multi-resolution Inception-ResNet (44) network architecture for the suppression of motion artefacts. We also demonstrate motion suppression for both simulation and experimental datasets, which were subsequently compared with an iterative entropy minimization technique. The proposed method, referred to as MocoNet, is a data-driven approach based on a CNN that learns to identify and suppress motion artefacts from images retrospectively.

\section{METHODS}

\section{Model of Motion artefact in MR imaging}

Motion during MR data acquisition manifests itself as artefacts in the reconstructed images. The nature of the artefacts found in structural scans depends on the magnitude of the motion 
and the time point at which it occurred. In this work, we have neglected the motion during read-out. Consider a movement (i.e. position change) occurs at the time point $t$ during data acquisition (i.e. during the $\mathrm{n}^{\text {th }}$ phase encoding step). The motion-corrupted k-space can be considered as a linear combination of two partial sets of k-spaces (Fig.1 (c), (l)) before and after motion occurrence. The corresponding motion-corrupted image (Fig. 1 (m)) can then be modelled by a linear combination of motion-free images (before and after change of position, Fig. 1 (d, g)) convolved with a sampling kernel (Fig. 1(e, h)). Mathematically, the motioncorrupted image can be represented as:

$$
I_{m}^{k}=\sum_{t=0}^{t=T} I_{t}^{k} \circledast h_{t}=\sum_{t=0}^{t=T}\left(C_{k} M_{t} I_{0}\right) \circledast h_{t}
$$

where, $I_{m}^{k}$ is the motion-corrupted image from the $k^{t h}$ channel, $C_{k}$ is the coil sensitivity map, $\mathrm{T}$ is the number of times the subject moved, $I_{t}^{k}$ is the object (after motion) at time point $t, I_{0}$ is the motion-free image, $M_{t}$ is the motion matrix at time $t$, $\circledast$ is the convolution operation and $h_{t}$ is the convolution kernel determined by the time point $t$. The individual images from multiple channels can be combined to generate single motion corrupted image $I_{m}$ as:

$$
I_{m}=\left|\sum_{k=1}^{k=N_{c h}} C_{k}^{*} I_{m}^{k}\right|=\left|\sum_{k=1}^{k=N_{c h}} C_{k}^{*} * C_{k} \sum_{t=0}^{t=T}\left(M_{t} I_{0}\right) \circledast h_{t}\right|
$$

where, $C_{k}^{*}$ is a conjugate of coil sensitivity and $N_{c h}$ is the number of channels. Inspired by the similarity between the convolutional motion model presented in Eq.2 and CNN, we investigated the use of deep learning CNN to solve the motion correction problem. In order to estimate the motion-free image $I_{0}$ in Eq.2, the training process was applied to estimate a deep learning model that can identify and suppress the motion artefacts.

\section{Deep Learning Network Architecture}

The distribution of motion artefacts within the image is both local and global. The distribution of local artefacts varied within the different brain regions, with the cortical region of the brain having comparatively more artefacts than the mid-brain. The motion also resulted in ringing artefacts in the whole brain including outside the brain region in the background, 
which we consider as global artefacts. Since the neural network needs to learn both the global and local artefacts at multiple resolution scales, we designed a multiple resolution approach where the architecture in the encoder consisted of parallel paths with different convolution kernel sizes. The different kernel sizes results in a different effective field of view at each resolution scale.

Specifically, we used Inception ResNet-V2 architecture for the encoder network, which consisted of parallel paths with different convolutional kernel sizes. Since the depth of the complete encoder-decoder architecture was large, we also used a residual connection (45), as this only need to learns a residual function between the skip connected layers, resulting in faster training and convergence (44). Residual blocks are the concatenation connection from the last layer of the encoder to the first layer of the decoder at the same scale. The net effect of the residual connection is that the network between the residual connections only learns the residual function instead of the complete function. In the context of MR image reconstruction, the network block between the residual connections only has to learn the difference between an artefact image and a clean image instead of learning to reconstruct a completely clean image.

An encoder-decoder architecture (Fig. 2 (a)) was trained for motion correction. The encoder network consisted of an Inception-ResNet-V2 (44) architecture, and pre-trained weights trained on ImageNet dataset (46) were used for the initialization of the encoder part of the encoder-decoder network. The inception architecture (Fig. 2 (b)) consists of parallel convolution pathways with different convolutional kernel sizes. The use of different kernel sizes results in different receptive fields, giving rise to multi-resolution feature maps. The decoder is made up of a series of convolution and up-sampling layers (Fig. 2 (c)); two convolutions took place before every up-sampling operation. The decoder network includes dropout layers with a dropout fraction of 0.2 after every convolution to avoid any overfitting. The stride of one was used for all the convolution layers and a stride of two for the upsampling layers in the decoder. Skip connections (concatenation) were made between the same scale feature maps from the encoder and decoder part of the network. All the feature maps at a particular scale in the encoder were concatenated to the first layer of decoder with the same scale. The loss function used to train the network was the mean absolute error 
between the output image and the target image (no motion image). The input to the network during the training was a $256 \times 256$ motion-corrupted image and the output was the motioncorrected image. During the inference, the input image of arbitrary size can be first zero padded to the nearest multiple of 32 and can be used as input to the network.

\section{Motion Simulation}

Motion simulation was performed with randomly generated 3D rigid body motion between $\pm 5 \mathrm{~mm}$ of translation and $\pm 5^{0}$ of rotation on all three axes. The simulated motion range was consistent with a previous in-vivo cohort study (47). The MPRAGE images of size $256 \times 256 \times 256$ were used for simulation purposes and the motion was simulated along the phase encode direction (anterior-posterior). Any motion within a single phase encode was neglected in the simulations. The process of motion simulation is depicted in Fig. 3. The 3D image was rigidly transformed based on the six motion parameters (three translations and three rotation parameters) for each time point (phase encode). The k-space $\left(N_{p e} \times \mathrm{N}_{\mathrm{fe}} \times \mathrm{N}_{\mathrm{se}}\right)$ corresponding to a rigidly transformed image was computed and $1 \times \mathrm{N}_{\mathrm{fe}} \times \mathrm{N}_{\mathrm{se}}$ k-space corresponding to the current time point (phase encode) was extracted and appended to the motion-corrupted k-space. The appending of the k-space blocks $\left(1 \times \mathrm{N}_{\mathrm{fe}} \times \mathrm{N}_{\mathrm{se}}\right)$ for all phase encodes constituted the motion-corrupted k-space data $\left(N_{p e} \times \mathrm{N}_{\mathrm{fe}} \times \mathrm{N}_{\mathrm{se}}\right)$. The motioncorrupted, simulated k-space is inverse Fourier transformed to generate motion-corrupted images. The anterior-posterior (A-P) direction was considered as phase encodes, the left-right (L-R) direction was considered as slice encodes and the head-feet (H-F) direction was considered as frequency encodes for all the motion simulations.

Two different motion scenarios were simulated: (i) abrupt motion: where the patient moves abruptly during the scan from time to time. We simulated up to 16 abrupt motion events during a single MPRAGE scan, the number of motion events ranging from 0 to 16 were selected using a uniform random number generator. An example of a motion curve is shown in Fig. 6. (ii) gradual motion: where the patient makes gradual movements during the scan. An example of the motion curve for gradual motion is shown in Fig. 7. 
In order to generate abrupt motion curves, a random number is generated for the number of motion events ranging between 1 and 16. Then, six motion parameters are randomly generated for each motion event which results in an abrupt motion curve as shown in Fig. 7. For gradual motion, curves for abrupt motion are generated and then each motion curve is passed through a Gaussian filter, resulting in smoothly varying motion curves as shown in Fig. 6.

\section{Data Preparation and Training}

3D MPRAGE datasets of 262 subjects were used to generate a training dataset, and 64 subjects were used to generate a validation dataset. Six different random motions were simulated for each subject, thus providing 1572 subjects for training and 384 subjects for validation. In order to generate motion-corrupted images, 3D k-space datasets of the images were distorted with a simulated 3D rigid body random motion as described in the 'Motion Simulation' subsection. Both the motion-corrupted image and the motion-free images were normalized to 0.5 mean and 1/6 standard deviation so that approximately $95 \%$ of the intensity values lie between 0 and 1 . After processing through the deep learning network, the output motion-corrected image was renormalized to its initial mean and standard deviation. The CNN was trained to suppress motion artefacts from individual 2D slices of the 3D MPRAGE dataset.

The following parameters were used for training the network in the Keras deep learning library with Tensorflow backend: Adam optimizer (48) with initial learning rate = 0.0001 , batch size $=4$, periodic decay $=0.96$ after every epoch, each epoch consists of 2500 batches i.e. 2500 weights update per epoch, and a total of 100 epochs on the NVIDIA Tesla V100 GPU. The training and validation losses are shown in Fig. 4.

\section{Experimental Data Acquisition}

In vivo experiments were performed on a Siemens Skyra 3T MRI scanner (Siemens Healthineers, Erlangen, Germany) with a maximum gradient strength of $45 \mathrm{mT} / \mathrm{m}$ and a maximum slew rate of $200 \mathrm{mT} / \mathrm{m} / \mathrm{ms}$. Informed consent was obtained from volunteers in accordance with the Institution's human research ethics policy. For experimental validation 
of the proposed motion correction technique, 3D MPRAGE datasets were acquired with $\mathrm{TE} / \mathrm{TR} / \mathrm{TI}=3 / 2000 / 900 \mathrm{~ms}$, matrix size $=256 \times 256 \times 192$, resolution $1 \mathrm{~mm}$ isotropic, flip angle $=9^{0}$, acquisition time of $8 \mathrm{~min} 32$ sec. Two separate scans were performed for each volunteer: one without motion, in which the volunteers were asked to remain still and the second with motion, in which the volunteers were allowed to make natural movements during the scan. A total of four volunteers were scanned in this manner. The reconstructed images from the default reconstruction pipeline of the scanner were processed with the trained MocoNet network, resulting in motion-corrected images. A structural similarity index (SSIM) was used as the quantitative metric for the evaluation of motion-corrected images.

\section{RESULTS}

Both the magnitude of the motion and the k-space location where motion occurred determine the severity and the appearance of the artefact in the resulting image. For example, motion occurring in the outer k-space (i.e. high-frequency components) manifests as ringing in the reconstructed image (Fig. 5 (a-d)). Motion occurring near to the centre of the k-space (i.e. low-frequency components) manifests as blurring (Fig. 5 (e-h)). When the magnitude of the motion was increased while the motion occurring k-space location remained fixed (shown in Fig. 5 (a-d)), the ringing increased. In contrast, when the motion occurring k-space location was varied and the magnitude of the motion remained fixed, the blurring artefacts increased as the location of the motion in k-space moved towards the centre (shown in Fig. 5 (e-h)). From this simulation, it can be inferred that the location of the motion in k-space largely determines the appearance of the motion artefacts (ringing or blurring) and the magnitude of the motion largely determines the spatial distribution and severity of the motion artefacts.

Although the network was trained in the range of $\pm 5 \mathrm{~mm}$ translation and $\pm 5^{0}$ rotation, the predictions were performed on a wider range $\left( \pm 8 \mathrm{~mm}\right.$ and $\left.\pm 8^{0}\right)$ to test the generalization capability of the network to unseen cases. The results of the motion artefact suppression are shown in Figures 6 and 7 for gradual motion and abrupt motion scenarios, respectively. In order to assess the performance of the deep learning method, the results were compared with the entropy minimization method (27), which is also a post-processing based method. From 
Figures 6 and 7, we can see that the ringing artefacts were more effectively suppressed with the MocoNet, compared to the entropy minimization method, and that the SSIM indices were higher for the MocoNet reconstructions. It is evident from the enlarged images in Figures 6 and 7 that the motion artefacts were successfully removed using the MocoNet. In contrast, the entropy minimization method resulted in residual ringing, particularly in abrupt motion scenario. The entropy minimization method performed better for the gradual motion compared to the abrupt motion. However, the MocoNet outperformed the entropy-based method in both of the motion scenarios in terms of the SSIM index and normalised root mean squared error. Furthermore, the performance of the MocoNet was also found to be consistent in both gradual motion and abrupt motion simulations. The consistent performance of the MocoNet highlights that it is robust to both gradual and abrupt motion scenarios. The mean of SSIM index for the corrected images was improved from 0.9058 to 0.9338 and the mean of normalised root mean squared error decreased from 0.1993 to 0.1336 .

Fig. 8 shows the performance of the motion correction with varying levels of motion on the validation dataset. Artefacts are shown to increase from left to right. In all the cases, the MocoNet was able to substantially remove the artefacts from the motion-corrupted images, resulting in corrected images with higher SSIM and lower normalised root mean squared error. The improved delineation between white and grey matter is clearly noticeable in the enlarged motion-corrected images Fig. 8 (b, c) compared to the motion-corrupted images. As shown in Fig. 9, using a paired t-test p value $<0.001$, which was calculated on 384 validation subjects, the SSIM statistical parameters (median, $25^{\text {th }}, 75^{\text {th }}$ and span) for MocoNet corrected images improved compared to the uncorrected images. Likewise, a similar trend was found to be true for normalised root mean squared errors with a paired t-test $\mathrm{p}$ value $<0.001$.

The proposed MocoNet correction was also evaluated on the experimental datasets acquired as described in the 'Experimental Data Acquisition' subsection. The motioncorrupted DICOM images were processed with the MocoNet, which suppressed the motion artefacts in the images. Motion-corrected images from two volunteers for all three orthogonal slices are shown in Figures 10 and 11, along with the structural similarity index w.r.t to the no-motion image. Visual inspection of the enlarged images shows that the images corrected 
using the MocoNet method have substantially reduced motion artefacts compared to the uncorrected images. The SSIM index demonstrates the improvements in image quality as a result of using MocoNet. The mean SSIM for subject 1 was increased from 0.9012 to 0.9396 and for subject 2, it was increased from 0.8330 to 0.8895 . Similarly, the mean normalised root mean squared error was decreased from 0.1021 to 0.0759 for subject 1 and from 0.0976 to 0.0774 for subject 2 .

We also compared the computation time of the different correction methods. The entropy minimization is an iterative algorithm and thus the computation time is large. For the image size of $256 \times 256 \times 150$, the execution time of the entropy minimization method was 12 hours 46 minutes (46000 secs) with a CPU, while it was only 185 secs for the MocoNet method - a 250x speed up in execution time. The implementation of the MocoNet on a GPU Nvidia-V100 further reduced the execution time to 15 secs, making its use feasible in realtime clinical applications.

\section{DISCUSSION}

We have demonstrated that the motion artefacts in MR images can be modelled as a linear combination of convolution between transformed images and convolution kernels. This convolution motion model forms the basis of using CNN to learn a model that can correct for motion artefacts. Simulation results confirmed that motion at high-frequency results in ringing, whereas motion at low-frequency results in blurring and ghosting. The encoder in the MocoNet separates the motion-corrupted images into features consisting of motion artefacts. The neuron in the decoder network only allows the motion free features to be integrated into the final motion-corrected images. Although the network was trained on simulated motioncorrupted images without considering any spin history effect, the network was able to correct for the real motion, demonstrating the robustness of the MocoNet method.

Current state-of-the-art motion correction methods that rely on the image or k-space navigators interfere with the MR protocol to the detriment of contrast, scan time, and SNR. Other methods based on external sensors need calibration and are complex to set up (49). The method presented in this work is an innovative solution to the problem of motion during MRI 
acquisitions. First, unlike navigators, it requires neither the development nor the modification of acquisition sequences or image reconstruction methods. Second, it does not interfere with MR protocol parameters such as T1/T2/TI/TE, scan time or contrast. Third, it does not require any external hardware devices, thus making it relatively inexpensive to implement.

We have presented a novel method based on deep learning that is capable of removing motion artefacts from motion-corrupted MR images. The proposed method was validated on both the simulation and the experimental datasets. We have demonstrated that the network trained with simulated motion was able to generalise and was able to correct for the real motion that occurred during a cohort of subjects that were scanned. Although the motion simulation model captures wide range of realistic motion scenarios, but MR signal variations that may occur due to spin wraps, magnetic field changes and signal loss caused by motion are not modelled. This may degrade the performance of the motion suppression in few real motion scenarios. Integrating the Bloch equation into motion model would lead to more accurate data generation and hence more robust motion suppression system. The proposed method can work independently without the need for any motion parameter acquisition setup. Alternatively, it can also be used as a complementary method with existing motion correction setups such as navigators and external devices.

Compared with the entropy-based technique, the MocoNet method is significantly more effective in removing motion artefacts. However, there are occasionally residual motion artefacts left, especially when motion occurred at low-frequency k-space locations. In order to make the motion correction more robust, a hybrid approach of combining the MocoNet with navigators could result in a better motion correction system. It is known that navigators are good at picking-up large motion efficiently, hence in a hybrid motion correction system, images can be prospectively or retrospectively corrected using navigators and the residual motion in the resulting images can be corrected with MocoNet. Another source of residual artefact could be the patient motion, which is widely different from the simulated motion case. Residual artefacts can also result from a different acquisition ordering of the phase/slice encodings that can be minimized by fine-tuning the network with dataset corresponding to the acquisition order. 


\section{CONCLUSION}

An efficient data-driven technique based on a CNN has been developed for MR motion correction without the need for external sensors or internal navigators. The network is based on the Inception-ResNet architecture and is fully trained using simulated motion. Here we have demonstrated the application of the technique to 3D T1 MPRAGE experimental datasets. The MocoNet is computationally efficient and the faster inference time makes it practical for routine clinical practice.

\section{Acknowledgement:}

We would like to thank Alexander Loktyushin (Max Planck Institute for Intelligent Systems)

for sharing the code for 3D motion correction using focus entropy criteria.

\section{References:}

1. Gupta MP, Shringirishi MM. Implementation of brain tumor segmentation in brain mr images using kmeans clustering and fuzzy c-means algorithm. International Journal of Computers \& Technology. 2013;5(1):54-9.

2. Godenschweger F, Kägebein U, Stucht D, Yarach U, Sciarra A, Yakupov R, et al. Motion correction in MRI of the brain. Physics in Medicine \& Biology. 2016;61(5):R32.

3. Andre JB, Bresnahan BW, Mossa-Basha M, Hoff MN, Patrick Smith C, Anzai Y, et al. Toward quantifying the prevalence, severity, and cost associated with patient motion during clinical MR examinations. Journal of the American College of Radiology. 2015;12:689-95.

4. Ehman RL, Felmlee JP. Adaptive technique for high-definition MR imaging of moving structures. Radiology. 1989;173:255-63.

5. Pipe JG. Motion correction with PROPELLER MRI: Application to head motion and free-breathing cardiac imaging. Magnetic Resonance in Medicine. 1999;42:963-9.

6. Zaitsev M, Zilles K, Shah NJ. Shared k-space echo planar imaging with keyhole. Magn Reson Med. 2001;45(1):109-17.

7. Nunes RG, Jezzard P, Behrens TE, Clare S. Self-navigated multishot echo-planar pulse sequence for highresolution diffusion-weighted imaging. Magn Reson Med. 2005;53(6):1474-8.

8. Fu ZW, Wang Y, Grimm RC, Rossman PJ, Felmlee JP, Riederer SJ, et al. Orbital navigator echoes for motion measurements in magnetic resonance imaging. Magnetic Resonance in Medicine. 1995;34:746-53.

9. Johnson PM, Liu J, Wade T, Tavallaei MA, Drangova M. Retrospective 3D motion correction using spherical navigator echoes. Magnetic Resonance Imaging. 2016;34:1274-82.

10. Welch EB, Manduca A, Grimm RC, Ward HA, Jack CR. Spherical navigator echoes for full 3-D rigid body motion measurement in MRI2001.

11. Costa AF, Petrie DW, Yen YF, Drangova M. Using the axis of rotation of polar navigator echoes to rapidly measure 3D rigid-body motion. Magnetic Resonance in Medicine. 2005;53:150-8.

12. Petrie DW, Costa AF, Takahashi A, Yen YF, Drangova M. Optimizing spherical navigator echoes for threedimensional rigid-body motion detection. Magnetic Resonance in Medicine. 2005;53:1080-7. 
13. Liu J, Drangova M. Rapid six-degree-of-freedom motion detection using prerotated baseline spherical navigator echoes. Magnetic Resonance in Medicine. 2011;65:506-14.

14. White N, Roddey C, Shankaranarayanan A, Han E, Rettmann D, Santos J, et al. PROMO: Real-time prospective motion correction in MRI using image-based tracking. Magnetic Resonance in Medicine. 2010;63:91-105.

15. Thesen S, Heid O, Mueller E, Schad LR. Prospective Acquisition Correction for head motion with imagebased tracking for real-time fMRI. Magnetic Resonance in Medicine. 2000;44:457-65.

16. Gallichan D, Marques JP, Gruetter R. Retrospective correction of involuntary microscopic head movement using highly accelerated fat image navigators (3D FatNavs) at 7T. Magnetic Resonance in Medicine. 2016;75:1030-9.

17. Volumetric navigators for prospective motion correction and selective reacquisition in neuroanatomical MRI, (2012).

18. Buschbeck RP, Yun SD, Jon Shah N. 3D rigid-body motion information from spherical Lissajous navigators at small k-space radii: A proof of concept. Magn Reson Med. 2019.

19. Schulz J, Siegert T, Reimer E, Labadie C, Maclaren J, Herbst M, et al. An embedded optical tracking system for motion-corrected magnetic resonance imaging at 7T. Magnetic Resonance Materials in Physics, Biology and Medicine. 2012;25:443-53.

20. Zaitsev M, Dold C, Sakas G, Hennig J, Speck O. Magnetic resonance imaging of freely moving objects: prospective real-time motion correction using an external optical motion tracking system. Neurolmage. 2006;31:1038-50.

21. Maclaren J, Armstrong BSR, Barrows RT, Danishad KA, Ernst T, Foster CL, et al. Measurement and Correction of Microscopic Head Motion during Magnetic Resonance Imaging of the Brain. PLoS ONE. 2012;7:e48088.

22. Qin L, Van Gelderen P, Derbyshire JA, Jin F, Lee J, De Zwart JA, et al. Prospective head-movement correction for high-resolution MRI using an in-bore optical tracking system. Magnetic Resonance in Medicine. 2009;62:924-34.

23. Ooi MB, Krueger S, Thomas WJ, Swaminathan SV, Brown TR. Prospective real-time correction for arbitrary head motion using active markers. Magnetic Resonance in Medicine. 2009;62:943-54.

24. Sengupta S, Tadanki S, Gore JC, Brian Welch E. Prospective real-time head motion correction using inductively coupled wireless NMR probes. Magnetic Resonance in Medicine. 2014;72:971-85.

25. Haeberlin M, Kasper L, Barmet C, Brunner DO, Dietrich BE, Gross S, et al. Real-time motion correction using gradient tones and head-mounted NMR field probes. Magnetic Resonance in Medicine. 2015;74:647-60.

26. Atkinson D, Hill DL, Stoyle PN, Summers PE, Keevil SF. Automatic correction of motion artifacts in magnetic resonance images using an entropy focus criterion. IEEE Transactions on Medical imaging. 1997;16(6):90310.

27. Loktyushin A, Nickisch H, Pohmann R, Scholkopf B. Blind retrospective motion correction of MR images. Magn Reson Med. 2013;70(6):1608-18.

28. Quan TM, Nguyen-Duc T, Jeong W-K. Compressed Sensing MRI Reconstruction with Cyclic Loss in Generative Adversarial Networks. arXiv preprint. 2017:1-10.

29. Yang G, Yu S, Dong H, Slabaugh G, Dragotti PL, Ye X, et al. DAGAN: Deep De-Aliasing Generative Adversarial Networks for Fast Compressed Sensing MRI Reconstruction. IEEE Transactions on Medical Imaging2017.

30. Jin KH, McCann MT, Froustey E, Unser M. Deep Convolutional Neural Network for Inverse Problems in Imaging. IEEE Transactions on Image Processing. 2017;26:4509-22.

31. LeCun YA, Bengio Y, Hinton GE. Deep learning. Nature. 2015;521:436-44.

32. Lee D, Yoo J, Ye JC. Deep residual learning for compressed sensing MRI2017.

33. Zhu B, Liu JZ, Cauley SF, Rosen BR, Rosen MS. Image reconstruction by domain-transform manifold learning. Nature. 2018;555:487-92.

34. Hammernik K, Klatzer T, Kobler E, Recht MP, Sodickson DK, Pock T, et al. Learning a variational network for reconstruction of accelerated MRI data. Magnetic Resonance in Medicine. 2018;79:3055-71. 
35. Litjens G, Kooi T, Bejnordi BE, Setio AAA, Ciompi F, Ghafoorian M, et al. A survey on deep learning in medical image analysis. Med Image Anal. 2017;42:60-88.

36. Sciarra AM, Hendrik; Speck, Oliver. Machine learning algorithms for detection of motion artifacts: a general approach2018.

37. Bilgic B, Cauley SF, Chatnuntawech I, Manhard MK, Wang F, Haskell M, et al., editors. Combining MR Physics and Machine Learning to Tackle Intractable Problems. Proceedings of the 26th Annual Meeting ISMRM; 2018.

38. Haskell MW, Cauley SF, Bilgic B, Hossbach J, Splitthoff DN, Pfeuffer J, et al. Network Accelerated Motion Estimation and Reduction (NAMER): Convolutional neural network guided retrospective motion correction using a separable motion model. Magn Reson Med. 2019.

39. Johnson PM, Drangova M, editors. Motion correction in MRI using deep learning. Proc Intl Soc Mag Reson Med; 2018; Paris, France.

40. Johnson PM, Drangova M. Conditional generative adversarial network for 3D rigid-body motion correction in MRI. Magn Reson Med. 2019;82(3):901-10.

41. Küstner T, Armanious K, Yang J, Yang B, Schick F, Gatidis S. Retrospective correction of motion-affected MR images using deep learning frameworks. Magnetic resonance in medicine. 2019.

42. Pawar K, Chen Z, Shah NJ, Egan G, editors. Motion Correction in MRI using Deep Convolutional Neural Network. Proc Intl Soc Mag Reson Med; 2018.

43. Sommer K, Brosch T, Wiemker R, Harder T, Saalbach A, Hall CS, et al. Correction of motion artifacts using a multi-resolution fully convolutional neural network. Proceedings of the 26th Annual Meeting of ISMRM, Paris. 2018.

44. Szegedy C, loffe S, Vanhoucke V, Alemi AA, editors. Inception-v4, inception-resnet and the impact of residual connections on learning. Thirty-First AAAI Conference on Artificial Intelligence; 2017.

45. He KM, Zhang XY, Ren SQ, Sun J. Deep Residual Learning for Image Recognition. Proc Cvpr leee. 2016:7708.

46. Deng J, Dong W, Socher R, Li L-J, Li K, Fei-Fei L, editors. Imagenet: A large-scale hierarchical image database. 2009 IEEE conference on computer vision and pattern recognition; 2009: leee.

47. Chen Z, Sforazzini F, Baran J, Close T, Shah NJ, Egan GF. MR-PET head motion correction based on coregistration of multicontrast MR images. Human brain mapping. 2019.

48. Kingma DP, Ba J. Adam: A method for stochastic optimization. arXiv preprint arXiv:14126980. 2014.

49. Maclaren J, Aksoy M, Ooi MB, Zahneisen B, Bammer R. Prospective motion correction using coil-mounted cameras: cross-calibration considerations. Magnetic resonance in medicine. 2018;79(4):1911-21. 


\section{Figure Captions:}

Figure 1: Convolution motion model: showing that the motion-corrupted image can be modelled as a linear combination of convolution of rigidly transformed, fully sampled images $(d, g)$ and convolution kernels $(e, h)$. (a): $k$-space of fully sampled image; (b): $k$-space sampling mask (yellow represents 1 and blue represents 0); (c): partial $k$-space of the object; (d): object of interest; (e): convolution kernel determined by the sampling mask (b); (f): image formed by partial k-space; (g): object of interest rotated by $\Delta \theta$ and translated by $\Delta x$ and $\Delta y$; (j-l), $(\boldsymbol{h}-\mathbf{i})$ is same as (a-c), (e-f) respectively for the rotated and translated object (g); (m): the motion-corrupted image.

Figure 2: Network Architecture: (a): Encoder-Decoder convolution neural network architecture for motion correction. The encoder is Inception ResNet-V2 architecture and the decoder is a stack of convolution and upsampling operations; (b): Inception ResNet-A block in the encoder consisting of parallel paths with different convolution kernel size; (c): A block of convolution and upsampling in the decoder, six such blocks are used to form the decoder network; (d): description of the symbolic representation used in the figure. The input to the network is a 256x256 motion-corrupted image and output is a clean motion-corrected image, mean absolute error was used as a loss function to train the network.

Figure 3: Flow chart of motion-corrupted data generation process; $N_{p e}$ : number of phase encodes; $N_{f e}$ : number of frequency encodes; $N_{s e}$ : number of slice encodes. It consists of rigid body transformation of 3D MR image based on six motion parameters at given time, followed by the extraction of $1 \times N_{f e} \times N_{s e} k$-space lines, which are appended for all time points, resulting in $N_{p e} \times N_{f e} \times N_{s e}$ motion-corrupted $k$-space.

Figure 4: Plot of training and validation loss for the network trained with periodic learning rate decay and the adam optimizer. Each epoch consists of 2500 iterations.

Figure 5: Simulation demonstrating the effect of a single motion event on reconstructed images; Ref: represents reference image; (a), (b), (c) and (d) showing reconstructed images as a result of increasing the magnitude of the motion at a fixed phase encode $\left(100^{\text {th }}\right)$ in the acquisition process. The magnitude was increased as 1, 2, 4, and 6 times respectively; (e), (f), (g) and (h) show reconstructed images due to a fixed amount of motion at different phase encodes in the acquisition process. The phase encodes were $90^{\text {th }}, 100^{\text {th }}$, $110^{\text {th }}$ and $120^{\text {th }}$ respectively.

Figure 6: Simulated motion-correction results for gradual motion; plot in the top shows the simulated motion parameters where $R x, R y, R z$ are rotation in degrees, and $T x, T y, T z$ are translations in the $m m$ in $x, y, z$ directions, respectively. The $x$-axis represents time in terms of phase encodes (PE) and the time would be equal to PEXTR for the MPRAGE sequence. Reference: shows the motion-free clean image; Input: shows the image corrupted by the simulated motion; Entropy: image corrected using the entropy minimization method; Moconet: image corrected using deep learning method; NRMSE: normalised root mean squared Error; SSIM: structural similarity index.

Figure 7: Simulated motion-correction results for abrupt motion; plot in the top shows the simulated motion parameters where $R x, R y, R z$ are rotation in degrees and $T x, T y, T z$ are translations in $m m$ in the $x, y, z$ directions respectively. The $x$-axis represents time in terms of phase encodes (PE) and the time would be equal to PEXTR for the MPRAGE sequence. Reference: shows the motion free clean images; Input: shows the images 
corrupted by the simulated motion; Entropy: shows images corrected using the entropy minimization method; MocoNet: shows images corrected using the deep learning method. NRMSE: normalised root mean squared error; SSIM: structural similarity index.

Figure 8: Simulated motion-correction results at different levels of motion artefacts on the validation dataset, severity increasing from left (a) to right (c); no motion: reference image without any motion artefacts; with motion: image with simulated motion artefacts; MocoNet: images corrected with the proposed motion correction method. The number below the enlarged images shows the SSIM index and the numbers below the difference images show the normalised root mean squared error.

Figure 9: Structural similarity (SSIM) and normalised root mean squared error (NRMSE) for the motioncorrupted vs motion-corrected image; the top of the box represents the $25^{\text {th }}$ percentile, the bottom of box represents the $75^{\text {th }}$ percentile, the red line represents the median. The paired t-test $p$ value was $<0.001$ for both $S S I M$ and $R E$

Figure 10: Experimental results for volunteer-I, in which two scans were performed - one with motion and one without motion; no motion: images from the scan in which the volunteer did not move; with motion: images from the scan in which the volunteer moved randomly at his/her own discretion; MocoNet: images from the scan where the volunteer moved and the motion-corrupted images were subsequently corrected using MocoNet network; Entropy: images corrected with the entropy minimization method. The numbers below the enlarged images shows the SSIM index and the numbers below the difference images show the normalised root mean squared error.

Figure 11: Experimental results for volunteer-II, in which two scans were performed one with motion and one without motion; no motion: images from the scan in which the volunteer did not move; with motion: images from the scan in which the volunteer moved randomly at his/her own discretion; MocoNet: images from the scan where the volunteer moved and the motion-corrupted images were subsequently corrected using the MocoNet network; Entropy: images corrected with the entropy minimization method. The numbers below the enlarged images shows the SSIM index and the numbers below the difference images show the normalised root mean squared error.

\section{List of Abbreviations:}

$\begin{array}{ll}\text { DL } & \text { : Deep Learning } \\ \text { CNN } & \text { : Convolutional Neural Network } \\ \text { MocoNet } & \text { : Motion Correction Network } \\ \text { MR } & \text { : Magnetic Resonance } \\ \text { SSIM } & \text { : Structural Similarity } \\ \text { EPIK } & \text { : Echo Planar Imaging with Keyhole } \\ \text { PROPELLER } & \text { : Periodically Rotated Overlapping Parallel Line with Enhanced Reconstruction } \\ \text { SNR } & : \text { signal to noise ratio } \\ \text { FOV } & : \text { Field of View } \\ \text { Pe } & \text { : phase encodes } \\ \text { Fe } & : \text { frequency encodes }\end{array}$



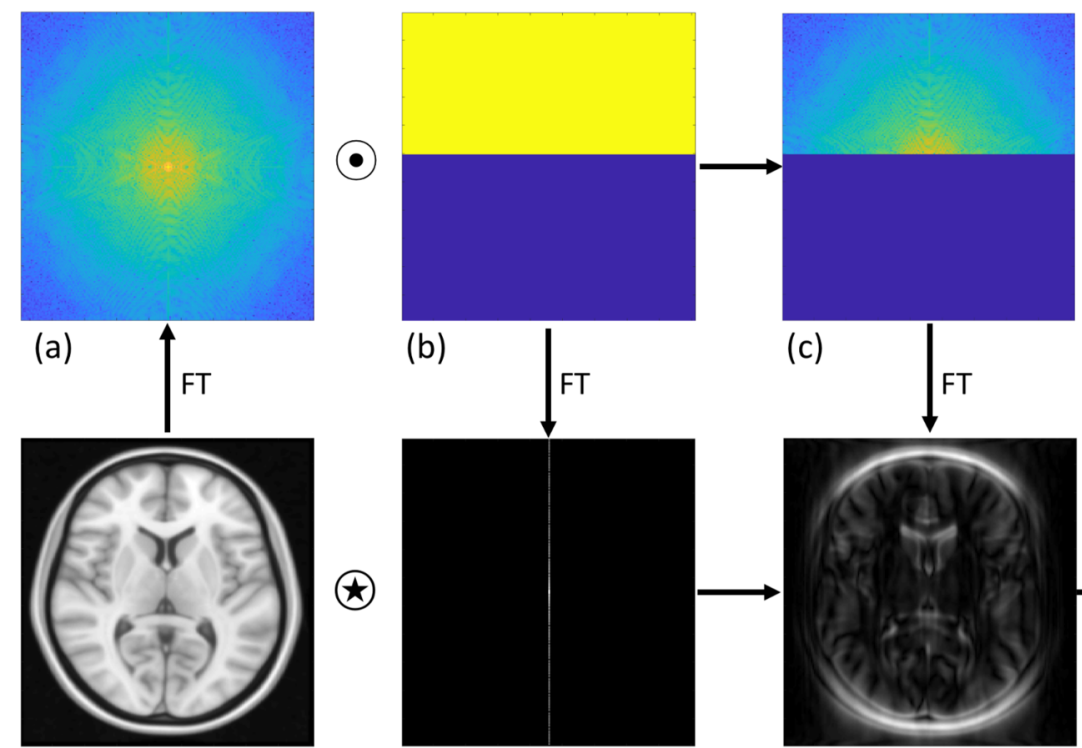

(d)

(e)
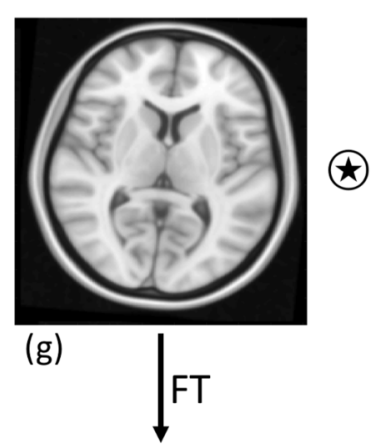

(h)

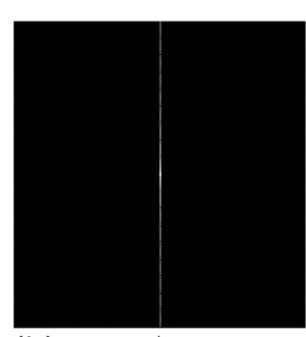

(f)
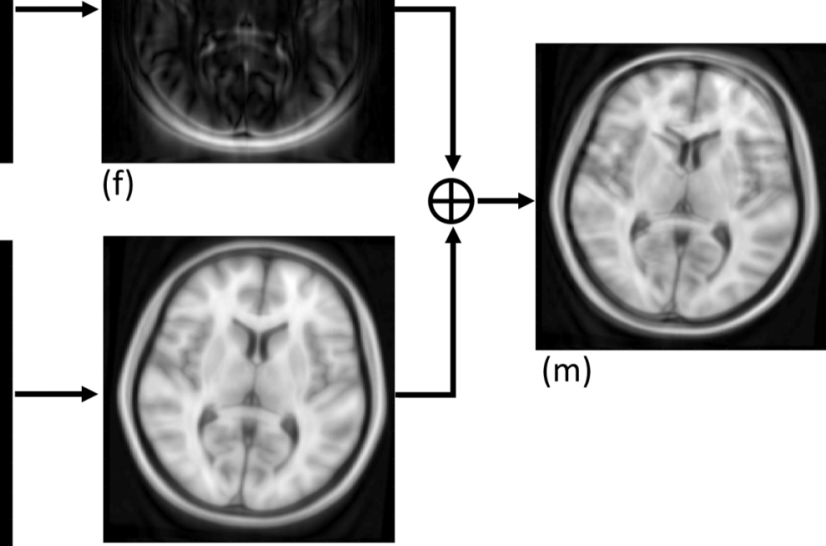

(m)
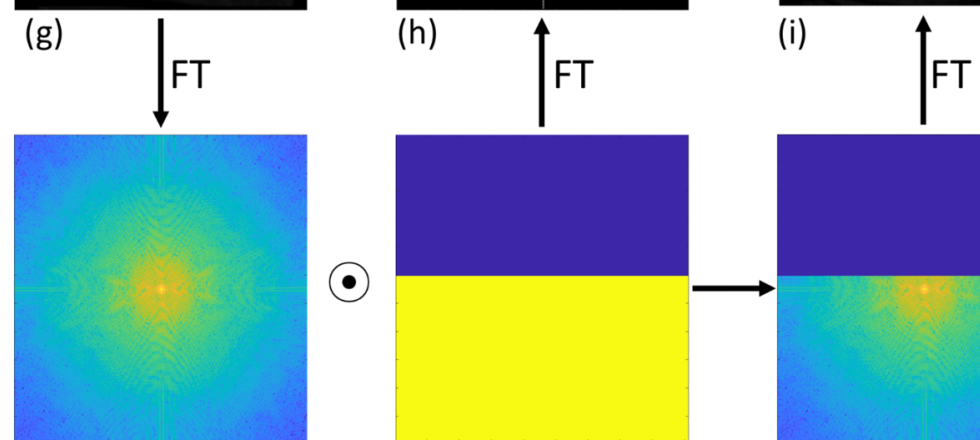

(j)

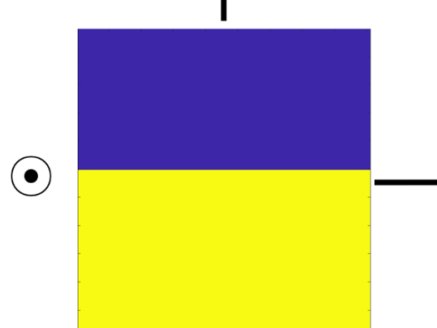

(k)

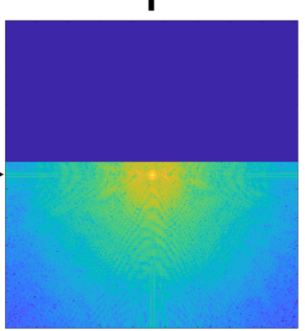

(I)

NBM_4225_Figure 1.tif

- : Pointwise multiplication

$\bigoplus$ : Pointwise addition

$\star$ : 2D convolution

FT : 2D Fourier transform 

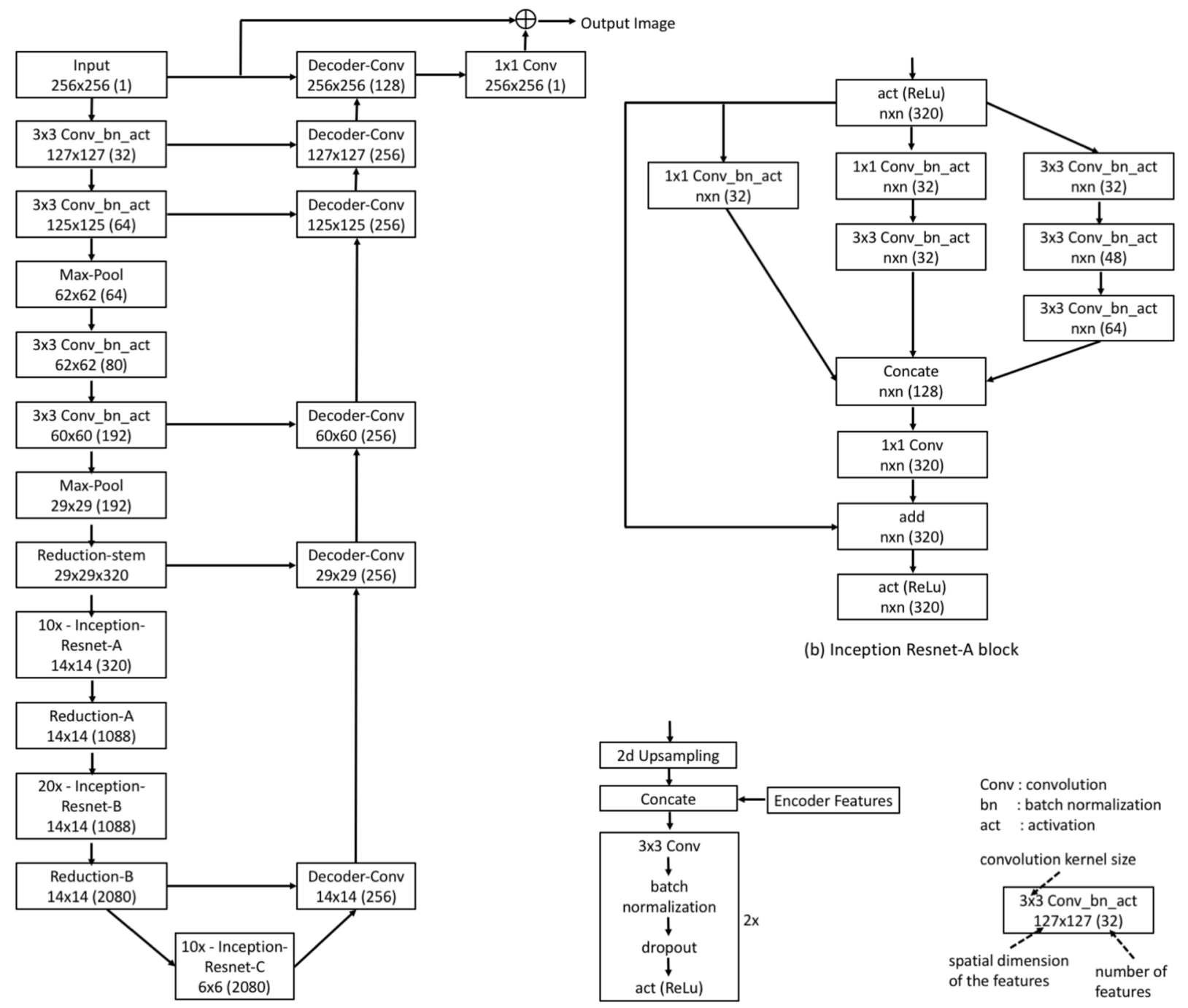

(b) Inception Resnet-A block

(a) Inception Resnet-v2 encoder-decoder architecture

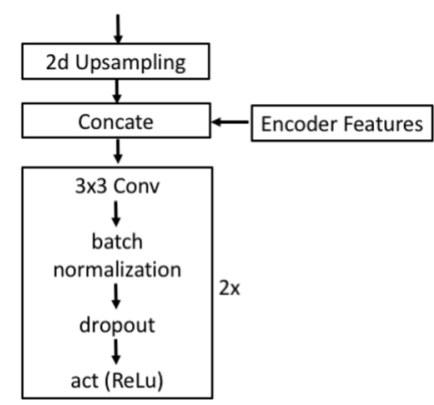

(c) Decoder-Conv

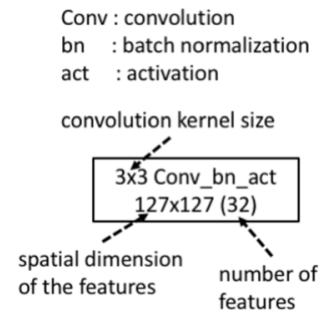

(d) Symbolic representations

NBM_4225_Figure 2.tif 


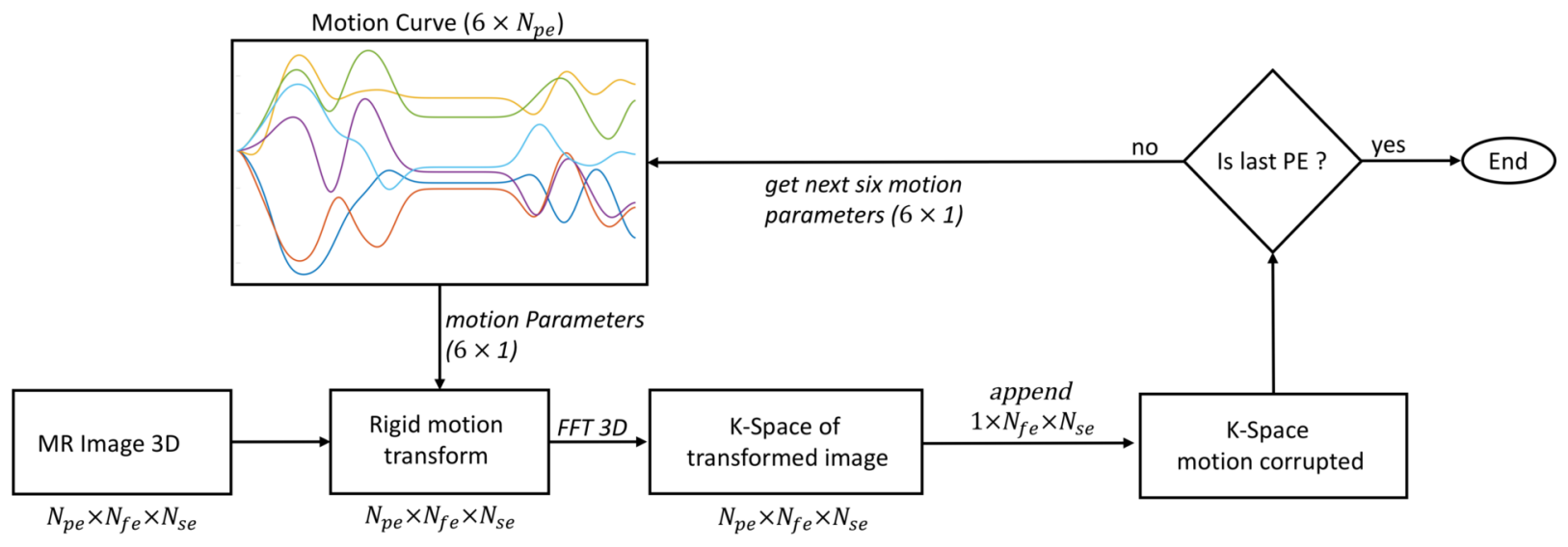

NBM_4225_Figure 3.tif

This article is protected by copyright. All rights reserved. 


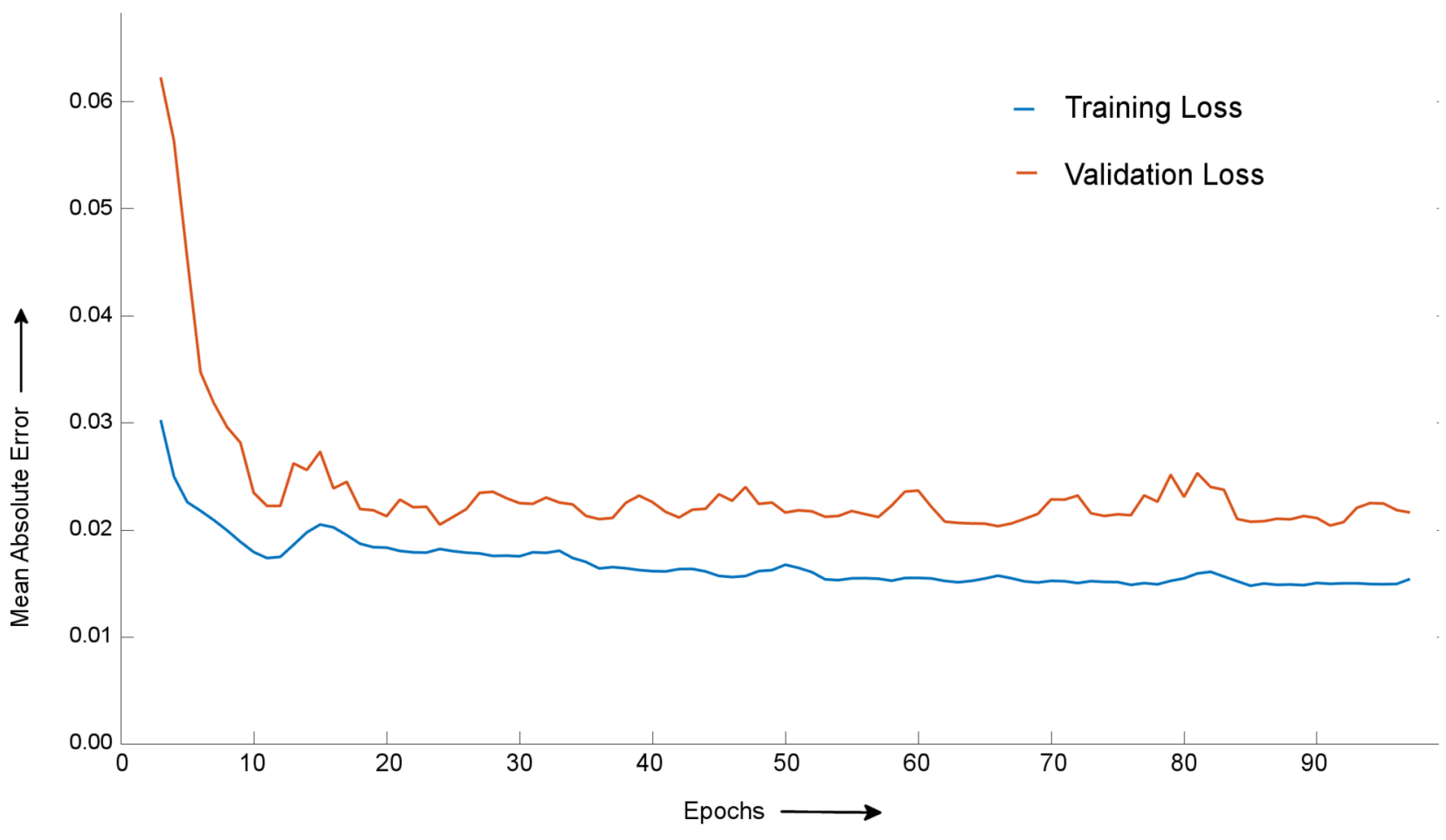

NBM_4225_Figure 4.tif

This article is protected by copyright. All rights reserved. 


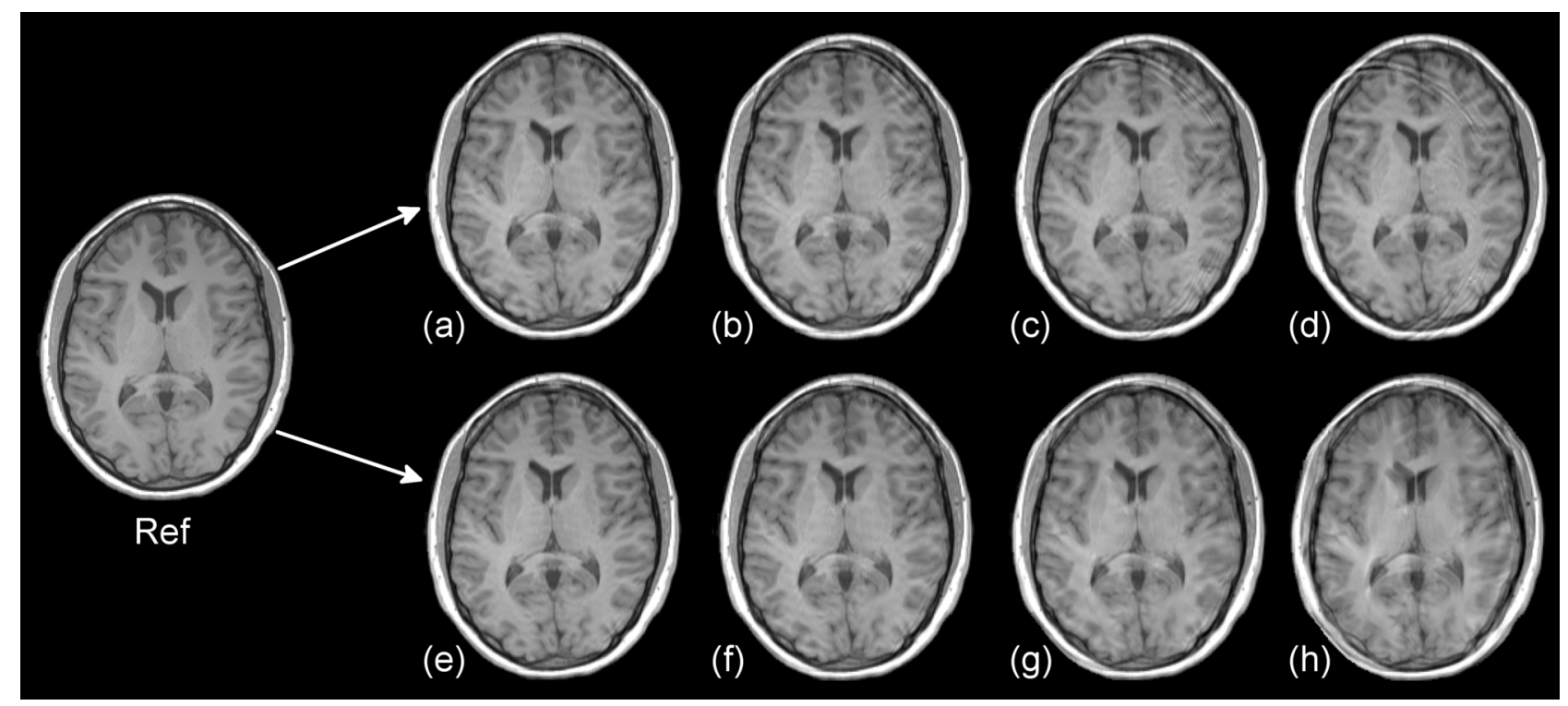

NBM_4225_Figure 5.tif

This article is protected by copyright. All rights reserved. 

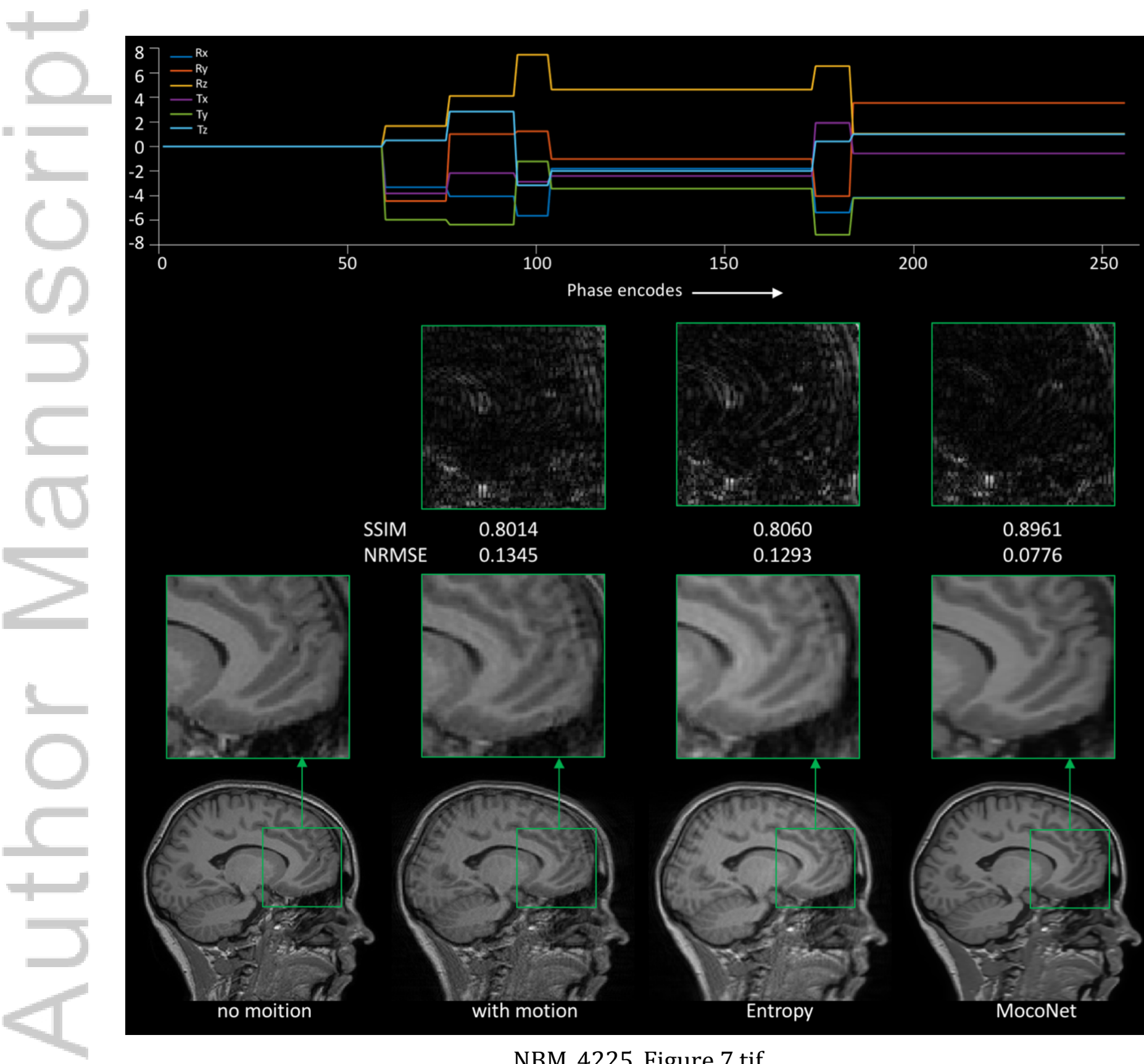

NBM_4225_Figure 7.tif

This article is protected by copyright. All rights reserved. 

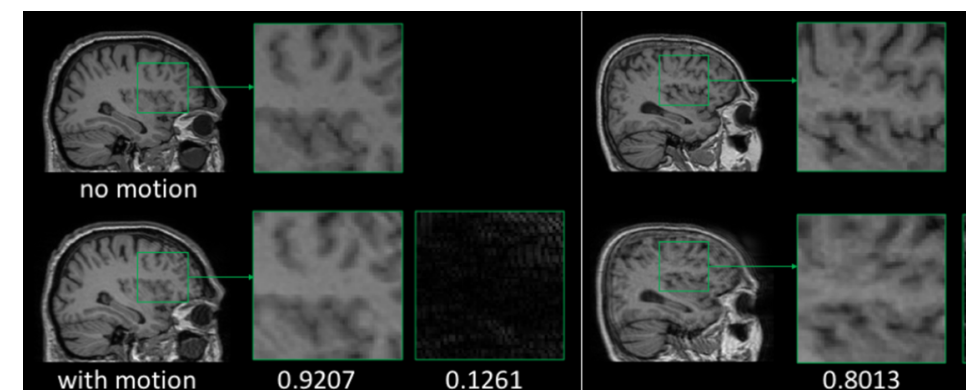

with motion

0.9207

0.1261
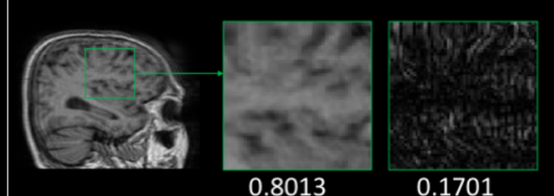

L.t.
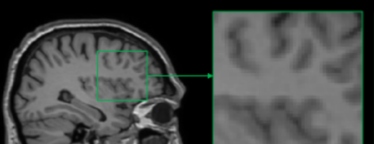

a) MocoNet

0.9324

0.1017

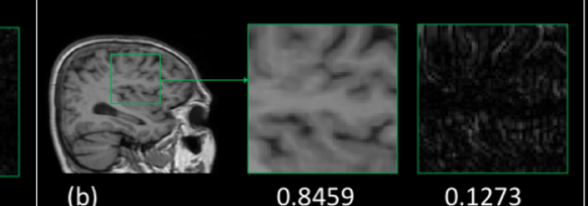

NBM_4225_Figure 8.tif

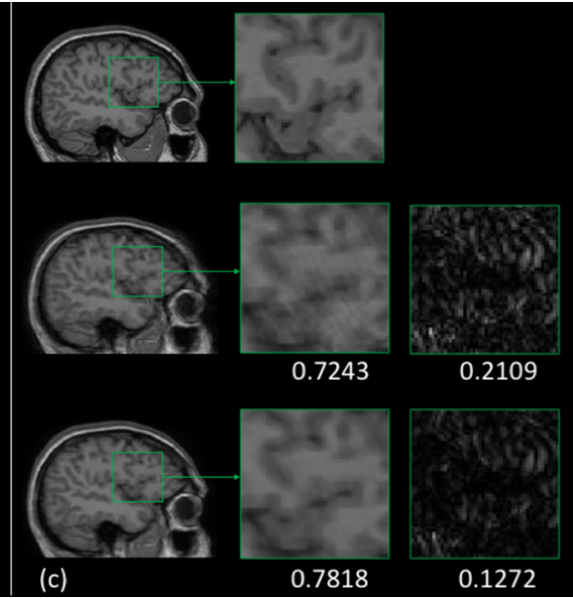

0.7818

This article is protected by copyright. All rights reserved. 

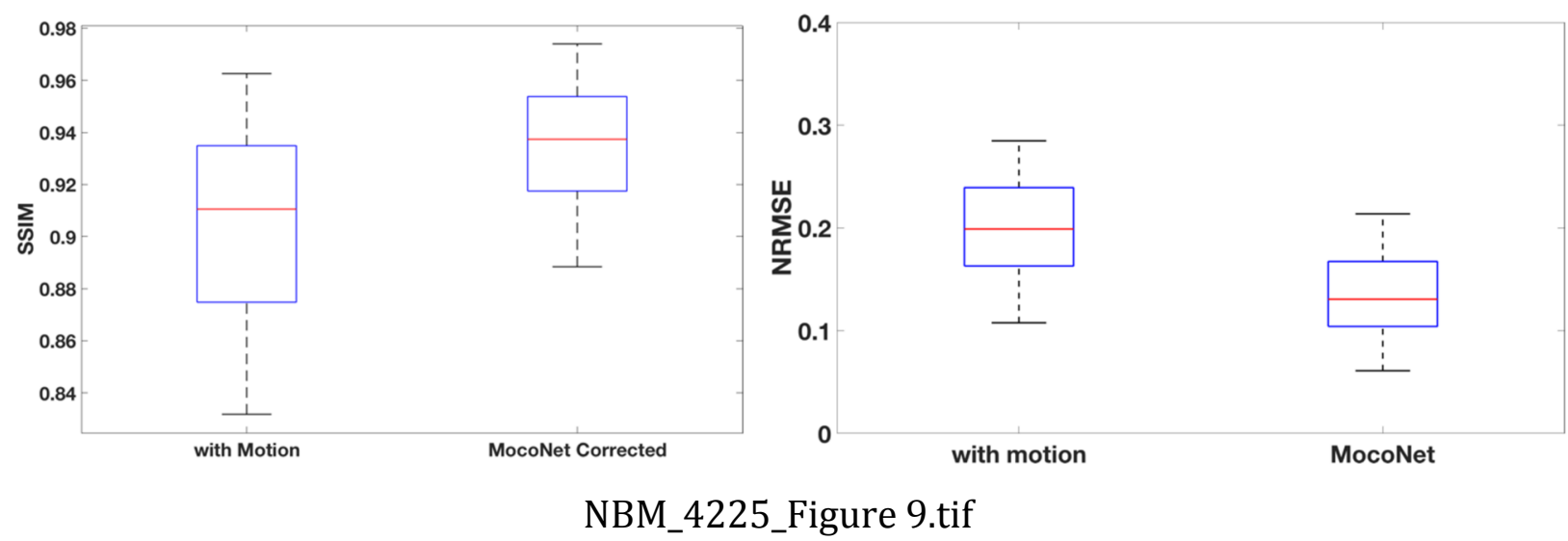

This article is protected by copyright. All rights reserved. 


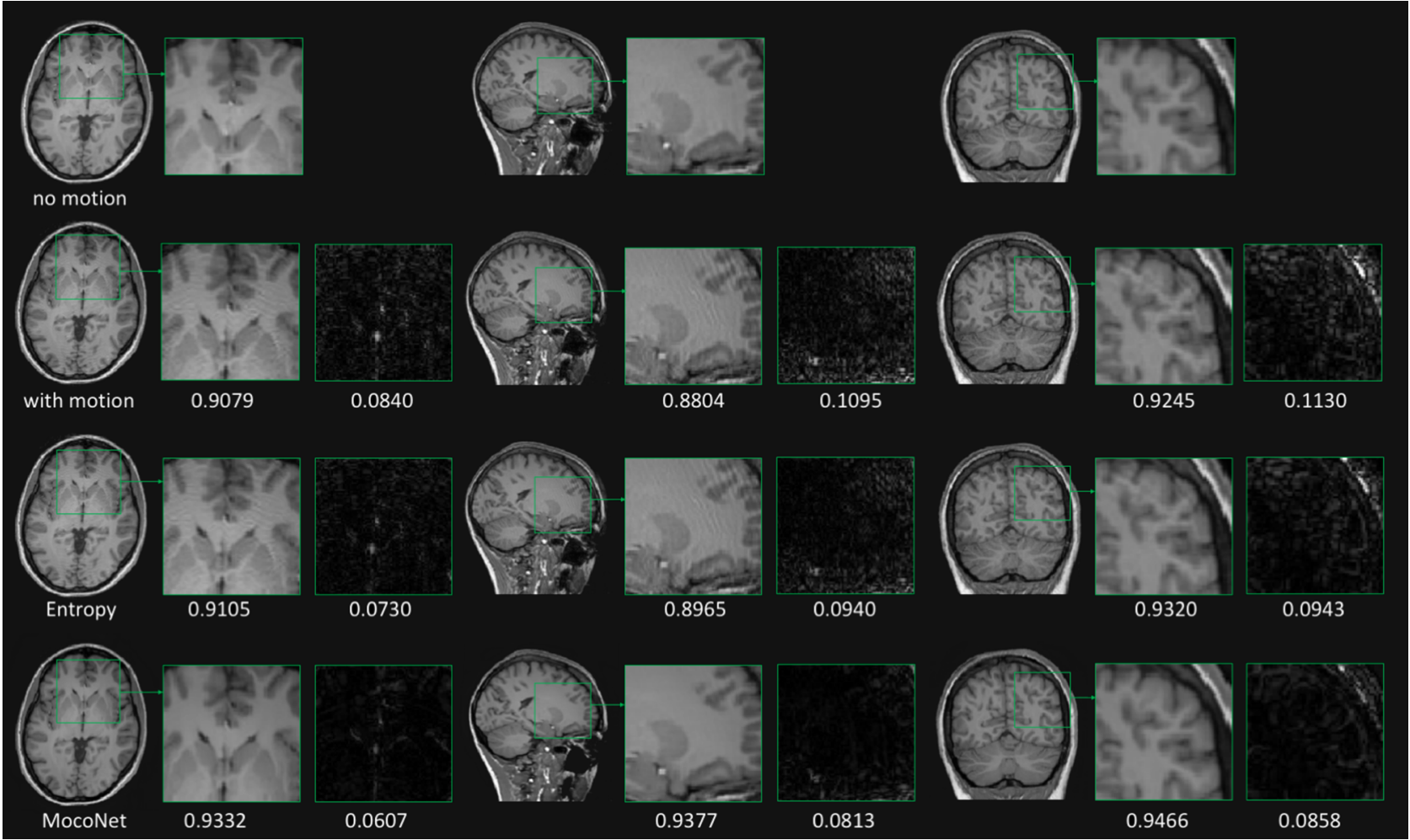

NBM_4225_Figure 10.tif 


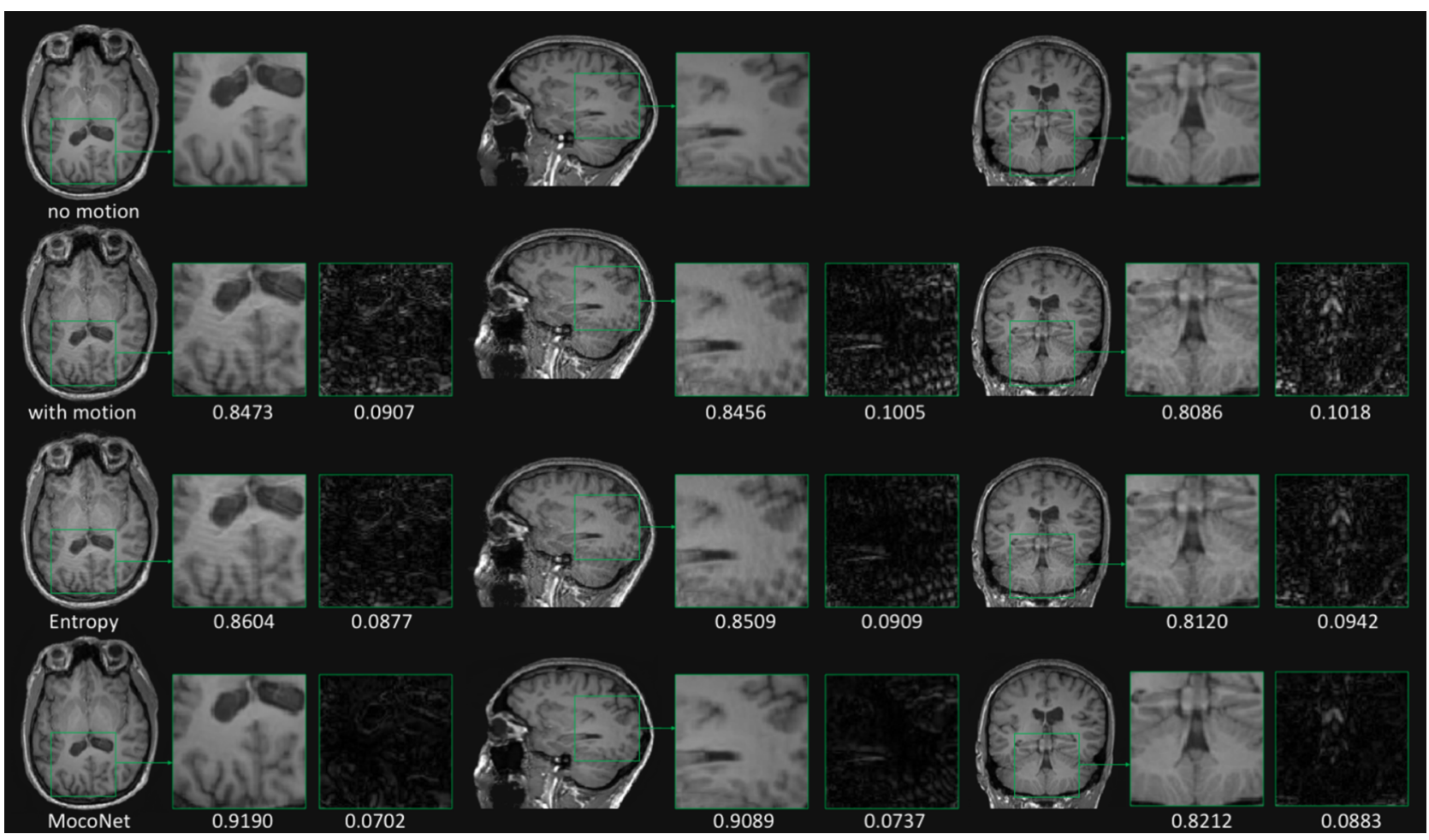

NBM_4225_Figure 11.tif

This article is protected by copyright. All rights reserved. 